\title{
La cooperación y la seguridad nacional en las relaciones entre Canadá y México
}

DOI: $10.32870 /$ mycp.v3i10.104

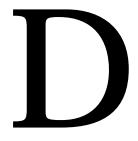
urante la historia contemporánea, la seguridad y la cooperación han sido temas controvertidos. En su sentido geográfico más amplio, estos términos algunas veces son utilizados para justificar, o encubrir, el expansionismo o la promoción unilateral de intereses particulares sobre los preceptos básicos de la ley internacional. De ahí la necesidad de tratarlos con mucha cautela, especialmente cuando se pretende abordar el derecho intrínseco de cada nación de garantizar su propia seguridad, así como la responsabilidad inherente de encontrar un equilibrio entre sus aspiraciones nacionales y sus compromisos internacionales.

Este hecho cobra una especial relevancia en el período posterior a la Guerra Fría, en que con la desintegración de la Unión Soviética y la extinción resultante del sistema bipolar ha llevado a una escenario internacional igualmente complejo. Estamos viviendo en un período de tensión dinámica entre fuerzas contradictorias - por un lado la tendencia hacia la integración económica como resultado de los procesos de globalización y, por otro lado, la tendencia hacia la fragmentación política y social, como resultado del resurgimiento del nacionalismo, los conflictos interétnicos y los fundamentalismos religiosos-. Pero lo más preocupante es que estas contradicciones han rebasado los mecanismos de que dispone la comunidad internacional para contener estos retos de la seguridad global.

Así, cada Estado ha enfrentado el imperativo de redefinir completamente su visión sobre la seguridad nacional, partiendo del innegable hecho de que la capacidad de decisión interna contiene hoy un inevitable componente internacional. Ningún país puede aislarse de

* Investigador del Departamento de Estudios del Pacífico de la Universidad de Guadalajara.
José Jaime López*

esta realidad, particularmente cuando la proximidad geográfica está combinada con la interdependencia económica.

Para muchos países, entre ellos Canadá y México, la soberanía representa el valor más importante de su nacionalidad. Su defensa y fortalecimiento constituye la meta principal del Estado. Precisamente, uno de los principales objetivos de su política exterior es el fortalecimiento de la capacidad para garantizar la seguridad nacional, junto con la promoción activa de sus intereses mediante la cooperación internacional y crecientes vínculos con los centros de la economía mundial. En este contexto, Canadá se ha convertido en un componente esencial de la estrategia de México para diversificar sus relaciones internacionales, en la medida en que representa un factor de equilibrio en sus relaciones con Estados Unidos.

Después de medio siglo de relaciones diplomáticas formales, caracterizadas por el respeto y la cordialidad, estas dos naciones hoy en día enfrentan un mundo en transición, lleno de retos pero también de oportunidades, en el cual ambos países tienen bases más sólidas para una rica y fructífera cooperación. De ser países distantes y mutuamente desconocidos, se han trasladado hacia una auténtica alianza estratégica que requiere basarse cada vez más en sus similitudes y complementariedades, así como en el respeto total de sus diferencias nacionales e individuales.

El hecho de pertenecer al mismo espacio geográfico y económico, de ser vecinos de la única superpotencia mundial y de su creciente identificación en varias áreas de la política internacional, han fortalecido la comunicación y las relaciones bilaterales en todos los ámbitos. El mejor conocimiento de uno y de otro ha contribuido también a esta relación, ambos países han aprendido a mirarse en forma directa 
y no únicamente, como en el pasado, a través del lente de su vecino común.

Sin duda, el Tratado de Libre Comercio de América del Norte (TLCAN) ha constituido el punto de partida de esta nueva etapa de acercamiento. Sin embargo, el surgimiento de la alianza estratégica mexicano-canadiense va más allá del comercio. La creciente agenda, rica y diversificada, cobra un aspecto bilateral, trilateral, regional y global. En este sentido, no es una exageración afirmar que en los últimos seis años, de todas las relaciones bilaterales de México, la establecida con Canadá ha experimentado el mayor crecimiento y dinamismo.

El innegable progreso realizado en este proceso de vinculación en beneficio de ambas naciones requiere redoblar los esfuerzos para consolidar e incrementar sus lazos y se establece una visión a futuro de sus prioridades. Con ese propósito, en la Reunión Ministerial, celebrada en abril de 1995, los dos gobiernos se comprometieron a elaborar la declaración de principios y objetivos que proporcionará la estructura y dirección de sus estrategias y acciones para el siglo XXI. En esta tarea, como en muchas otras, la participación activa de diversos representantes de la sociedad-incluyendo el sector privado, la comunidad académica y las organizaciones no-gubernamentales (ONG)-es de vital importancia.

Si bien es cierto que existe un gran número de metas comunes por alcanzar, también existen grandes diferencias en cuanto a enfoques y medios, específicamente las muy distintas visiones que los dos países tienen sobre las cuestiones de seguridad internacional, particularmente las relacionadas con la defensa y la promoción de la democracia en el mundo. Este tema es precisamente una prioridad indiscutible de la política exterior de Canadá.

El gobierno canadiense ha mantenido una postura de apoyo incondicional para toda la región y las iniciativas mundiales orientadas al establecimiento de mecanismos para la cooperación en la defensa de la democracia, su participación en las operaciones de pacificación de las Naciones Unidas son una muestra de ello.

Por su parte, México sostiene que la consolidación de los sistemas democráticos debe ser el resultado de procesos internos que, aunque apoyados desde el exterior, ante todo deben responder y reflejar aspiraciones legítimas de cada sociedad en el ejercicio de sus derechos de autodeterminación. Es importante reconocer que las diferencias en cuanto a los enfoques son una parte integral y natural de una relación como la que los mexicanos y canadienses están construyendo.

Quizás el reto más importante para Canadá y México es el seguimiento de los acuerdos resultantes de la Cumbre de las Américas, realizada en Miami en diciembre de 1994, en torno al mantenimiento y fortalecimiento de la democracia, la promoción de la integración económica y el libre comercio, la erradicación de la pobreza y de la discriminación en el hemisferio, el desarrollo sustentable y la conservación del ambiente.

Dentro de esta línea está el proyecto iniciado por Estados Unidos para aumentar la cooperación y el intercambio de información entre las fuerzas armadas en el continente. Debido a su importancia e implicaciones, los compromisos resultantes de la reunión de Ministros de Defensa de las Américas, realizada en julio de 1995 en Williamsburg, Virginia, también requieren de un profundo análisis y es en este campo Canadá y México pueden hacer una valiosa y constructiva contribución. Sin embargo, es esencial decidir si este ambicioso proceso será llevado al interior de la Organización de los Estados Americanos (OEA), o si se establecerán nuevos mecanismos institucionales para la cooperación regional.

Lo que es cierto es que el seguimiento del modelo que surja de Miami, la integración política y económica, jun to con la cooperación en el campo militar no pueden y no deben ser el resultado de decisiones unilaterales. Las diferencias políticas, económicas y sociales entre los países de la región y la diversidad de 
condiciones geoestratégicas requieren de un amplio proceso de consulta. En última instancia, la seguridad hemisférica es una derecho y responsabilidad de todos; no puede ser considerada por separado de las reglas básicas del derecho internacional. Tampoco la defensa de la democracia y la promoción de los derechos humanos, ni la lucha contra las grandes amenazas para la seguridad de muchos estados -como el tráfico de drogas, la corrupción, el contrabando de armas y el lavado de dinerojustifican acciones que violen los principios universalmente establecidos, tal como la soberanía, la no-intervención en los asuntos internos de los países y la auto-determinación de los pueblos. En resumen, la seguridad hemisférica es un objetivo común que requiere del apoyo de todo los países en la región; no puede estar basada en los intereses de algunos sobre los de otros.

En esta nueva etapa de relaciones y conocimiento mutuo entre México y Canadá, se observa que aspectos o temas que eran intocables ayer, hoy son ampliamente discutidos por sus gobiernos y sociedades, lo que constituye una prueba más de la madurez que las relaciones entre los dos países han alcanzado. Y, aún cuando este nuevo encuentro no ha estado exento de obstáculos y desavenencias, lo cierto es que los dos países están más unidos y se conocen mejor de lo que pudiera imaginarse hasta hace poco menos de una década. El factor fundamental de esto, por supuesto, ha sido la firma y entrada en vigor del TLCAN. En gran parte el crecimiento en la relación bilateral ha sido más que simplemente comercial, en los campos de la inversión, el cultural, el académico, el político y el diplomático. Por lo tanto, se puede afirmar que aún cuando el comercio tiende a acaparar la atención, con un crecimiento de más de 400 por ciento en los primeros años de operación del Tratado, forma parte de una relación más amplia.

\section{Evolución de la relación entre México y Canadá}

Como muchos observadores han señalado, no es que faltara mayor interés de Canadá hacia México, o de México hacia Canadá. Más bien, las dificultades en establecer una relación más cercana han estado siempre presentes, en tanto que los intentos por superarlas han sido esporádicos y frecuentemente poco entusiastas. Del mismo modo, la presencia y dominio de Estados Unidos en Norteamérica, y por el hecho de situarse en medio de los dos países, siempre tendió a impedir el acercamiento de sus dos vecinos. De manera paradójica, ahora es este país el que está forzando a los otros dos a caminar juntos.

Durante mucho tiempo, debido a factores políticos relacionados con la dependencia parcial de Canadá con el Reino Unido, hubo realmente pocas posibilidades de un acercamiento entre ambos países. En el campo diplomático, por ejemplo, la relación conflictiva entre Gran Bretaña y México dificultó la relación de Canadá con este último. Por otra parte, la permanente inestabilidad política de México también redujo cualquier posibilidad de Canadá para desarrollar un interés permanente sobre nuestro país. En efecto, las primeras misiones canadienses enviadas a Latinoamérica y el Caribe en 1865 para estudiar y fomentar el comercio con los países de la región, se vieron impedidas de visitar México debido a la guerra que libraba nuestro país contra los franceses. Tuvieron que pasar cuatro décadas para que la primera oficina comercial canadiense fuera abierta en México. 
Esto no significaba que la inversión canadiense -frecuentemente ligada con la de otras partes del Imperio y particularmente de la misma Gran Bretaña- no encontrara lugar en México. En realidad, los canadienses encontraron atractivo invertir su dinero en México en ferrocarriles, transporte urbano, electricidad, banca, puertos y, en cierta medida, en el petróleo. Sin embargo, Canadá vio con frustración, al igual que el resto del Imperio Británico, la caída de Porfirio Díaz en 1911 y las secuelas de la Revolución Mexicana. De igual forma, debido a las tensas relaciones existentes entre Londres y Washington durante gran parte del primer siglo de la independencia de Estados Unidos (en 1776), y las igualmente difíciles relaciones de México con Estados Unidos desde la independencia hasta la administración de Roosevelt en 1930, hubo pocos momentos en donde Gran Bretaña o Canadá sintieran la suficiente confianza para buscar el apoyo de México a fin de establecer un punto de equilibrio en su relación con Estados Unidos.

Así, por diversas razones, Canadá y México no desarrollarían una relación más estrecha (ni relaciones diplomáticas) hasta la llegada de la Segunda Guerra Mundial. Si en 1865 fue la amenaza de perder el mercado de Estados Unidos lo que empujó a los canadienses a mirar hacia los países del sur del continente, en la Segunda Guerra Mundial fue la pérdida de los mercados de Europa lo que obligó a Canadá a buscar nuevos mercados, entre ellos México. Fue de esta manera como el intercambio de ministros entre ambos países tuvo lugar en 1944.

Con el establecimiento de relaciones formales se lograron avances significativos, pero el fin de la guerra redujo la importancia de los vínculos debido a la rápida recuperación de los mercados de Europa. Con ello, Europa y Estados Unidos permanecieron en el centro de la atención de la política exterior de Canadá. Este hecho provocó que el interés mostrado en tiempos de la guerra por una Unión PanAmericana desapareciera, al menos de manera parcial, debido al rechazo de los estadounidenses de ver a los canadienses en esa organización durante el conflicto armado. La Guerra Fría no sólo forzó a Canadá a concentrar su atención y esfuerzos en sí misma, sino que también reafirmó las visiones estereotipadas existentes sobre Latinoamérica, y también sobre México.

México se benefició del retorno a la normalidad de los mercados internacionales, y su propia vinculación con Europa cobró fuerza nuevamente, siendo bajo estas circunstancias en que las relaciones entre Canadá y México perdieron terreno. Posteriormente, el Primer Ministro John Diefenbaker intentó diversificar el comercio de Canadá, dando un nuevo impulso a la relación comercial a fines de los cincuenta y principios de los sesenta, pero los vínculos fueron también esporádicos e insuficientes para alcanzar este objetivo.

Por su parte, los intentos de México por diversificar su comercio, en el contexto de la constante preocupación que los sucesivos gobiernos de nuestro país sentían por la excesiva dependencia de un solo mercado y fuente de apoyo -Estados Unidos-, incluían a Canadá como un objetivo junto con el resto del mundo. Estos intentos comenzaron después de la Segunda Guerra Mundial. En la administración de Adolfo López Mateos (1958-1964), se convirtieron en una prioridad, aumentando paulatinamente el interés por Canadá durante los gobiernos de Luis Echeverría Álvarez, José López Portillo y Miguel de la Madrid Hurtado.

Aún cuando ninguno de los dos países consideraba a su contraparte una prioridad en sus relaciones comerciales, la coincidencia de intereses en cuanto a la diversificación comercial era muy similar. Habría que resaltar que Canadá y México, compartían una actitud similar con respecto al multilateralismo en sus relaciones internacionales, como un elemento esencial para acceder a los mercados y la inversión de Estados Unidos y del mundo en general, un sentimiento de que podrían hacer algo para moderar la política de la Guerra Fría de Estados Unidos, y la necesidad de proteger sus recursos naturales. El interés hacia México se hizo más evidente con la visita del Primer Ministro Pierre Trudeau, en 1976, debido a factores políticos y al sostenido crecimiento 
económico de nuestro país. Ello sirvió para que, aunque a un nivel muy reducido, los políticos canadienses y mexicanos se conocieran mejor.

A pesar del crecimiento del comercio entre ambos países, después de ese año, se puede decir que la conexión se mantuvo tenue hasta 1982 , cuando estalló la crisis financiera de México, disipando gran parte del interés que se había ganado. $\mathrm{Y}$, no obstante los posteriores intereses de vinculación de los dos países, a medida que la década avanzó, el contexto económico nacional e internacional ensombreció todos los esfuerzos por construir una verdadera relación cercana. La globalización y la simultánea, en sentido opuesto, conformación de bloques económicos de los últimos años provocaron cambios importantes. Aunado a esas tendencias, hubo una notable recuperación económica de México a finales de los ochenta y principios de los noventa.

Una vez más, sin embargo, el papel de Estados Unidos en el nuevo contexto de las relaciones entre Canadá y México fue crucial. Por diversas razones, Washington empezó a percibir la necesidad de integrar un bloque económico propio, quizás en respuesta al espectacular éxito de la integración económica de Europa y las perspectivas de que algo similar ocurriera en Asia. Fue así como, a fines de los ochenta, se hicieron propuestas para la formación de un área de libre comercio CanadáEstados Unidos. Asimismo, en 1990, se lanzaría la Iniciativa de las Américas para formar un área de libre comercio que incluyera todo el continente americano.

Es importante subrayar que no todos en Canadá recibieron con buenos ojos el área de libre comercio con Estados Unidos. Incluso se llegó a afirmar que la derrota de los conservadores, del Primer Ministro Bryan Mulroney en las elecciones de 1993, fue una clara señal de protesta en contra del acuerdo y sus efectos inmediatos sobre la industria y el empleo de Canadá. Como quiera que haya sido, Canadá pronto se dio cuenta que Estados Unidos estaba en otra negociación bilateral de libre comercio, esta vez con México. Y, a pesar de su molestia, el gobierno canadiense fue obligado a unirse a esas negociaciones si es que no deseaba quedarse en desventaja con su vecino.

De esta forma, el fortalecimiento en las relaciones entre Canadá y México, provocado por el TLCAN, no puede ser visto realmente como el resultado de una iniciativa independiente promovida por los dos países para un mayor acercamiento entre ambas partes. En todo caso, fue en sí mismo un efecto secundario o algo derivado de los intentos desesperados por asegurar su participación en el mercado estadounidense y, al mismo tiempo, para evitar el creciente proteccionismo experimentado por Estados Unidos.

No hay duda de que la firma y puesta en marcha del TLCAN ha permitido que las relaciones bilaterales, no sólo comerciales, estén creciendo constantemente. Una muestra de ello es el hecho de que muchas universidades en México han creado nuevos programas de estudio sobre Canadá y, a su vez, los cursos sobre México se han multiplicado en Canadá. De igual forma, el hecho de que Canadá haya montado la más grande exposición comercial en el exterior en su historia, en la ciudad de Monterrey, México, en marzo de 1994. Cabe señalar que, de 1991 a 1994, la Embajada de Canadá en la ciudad de México registró un incremento en el intercambio comercial y económico de 15 a 34 por ciento. Del mismo modo, las visitas de alto nivel entre los dos países han cobrado una mayor importancia y los jefes de gobierno han realizado, aún en momentos difíciles, visitas a su contraparte. Por ejemplo, el Primer Ministro Jean Chrétien, en vez de viajar a Estados Unidos o a Gran Bretaña, visitó México en su primera visita oficial a otro país, en un gesto que no pasó desapercibido para los mexicanos.

Y, aún cuando la cobertura y presencia de la prensa en ambos países es más bien imperceptible y persisten muchos estereotipos -especialmente sobre nuestro país-, México es cada vez más "noticia” en Canadá y viceversa.

Como se pudo observar con el levantamiento de los zapatistas y los asesinatos políticos 
en 1994, el referéndum sobre Quebec y la crisis financiera mexicana de fines de 1994, la población de ambos países ahora está más atenta sobre lo que sucede en cada uno de ellos. Por supuesto que este proceso de conocimiento mutuo ha tenido altas y bajas. El levantamiento zapatista no hizo más que poner a México en una situación difícil precisamente en el momento en que el TLCAN entraba en operación. De igual forma, la inestabilidad política que acompañó a las elecciones presidenciales de 1994 y la crisis financiera de ese mismo año, despertaron muchas dudas entre los canadienses sobre las ventajas del acuerdo comercial. Peor aún, muchas organizaciones no-gubernamentales, en especial aquellas relacionadas con los derechos humanos, criticaron fuertemente al gobierno mexicano.

Así, a pesar de que las nuevas relaciones entre Canadá y México en esta nueva etapa no empezaron sobre ruedas, tienen mucho que ofrecer. Canadá no pierde la fe en México, y los mexicanos no mostraron tanto su malestar por las reacciones de los canadienses, quienes creyeron lo peor acerca de su nuevo socio. Canadá está consciente de que, tras la fuerte crisis financiera de México, nuestro país ha mostrado una gran capacidad de respuesta para hacer frente a sus problemas. A fin de cuentas, el TLCAN, a pesar de los serios cuestionamientos que ha recibido en los dos países, ha implicado más beneficios que perjuicios.

La interrogante que se ha presentado es si existe, o debería existir, una dimensión de seguridad en las relaciones entre Canadá y México. En vista de las muy distintas experiencias de los dos países en este campo, no es sorprendente que haya posturas muy diferentes. Hay quienes señalan que es algo inevitable de cualquier relación bilateral con Estados Unidos, debido a que los estadounidenses insistirán en introducirlo en cualquier acuerdo que firmen. Esto, por el interés extremo de México sobre su soberanía, implica serias dificultades.

Canadá y México ven la cooperación en materia de defensa y de seguridad en muy distintas formas, como reflejo de la ubicación histórica de cada país. Por parte de Canadá persiste el sentimiento, con frecuencia basado en el poco conocimiento de la realidad mexicana, que las relaciones civiles-militares en nuestro país son muy similares a las de los demás países de Latinoamérica y que, por lo tanto, es mejor no tocar este campo en su relación con México. Esta posición se vio reforzada por las críticas que recibieron las fuerzas de seguridad mexicanas por su intervención en Chiapas contra los zapatistas en 1994.

Los argumentos a favor sugieren que, a fin de profundizar las relaciones entre Canadá y México, un área de tanto interés común como la seguridad no debería quedar excluida. Después de todo, este argumento sostiene que las percepciones canadienses y mexicanas, en relación con el desarrollo de tratados de seguridad tradicional y no-tradicional, son muy similares, en virtud de que estos países ven el tema de la no-proliferación en forma muy similar y han sido muy activos en los foros multilaterales sobre este tema. De igual manera, perciben el reforzamiento de la paz en Centroamérica en forma similar. El impacto sobre la seguridad regional de un mal manejo de la transición cubana es también un tema de interés que comparten Canadá y México. Esto ha quedado de manifiesto en las reuniones de discusión a altos niveles diplomáticos y en sus posturas sobre estos temas en la Organización de las Naciones Unidas y en la Organización de Estados Americanos.

De igual forma, a pesar de que el gobierno mexicano considera la lucha contra el tráfico de drogas como un tema de seguridad, a diferencia de los canadienses, no significa que los dos países no tengan un punto de vista similar sobre este tema. En realidad, la cooperación en esta área ya ha sido objeto de acuerdos entre los dos países. Mientras que los pacifistas son vistos por muchos en México como una amenaza para la soberanía nacional y una seria intromisión potencial en los asuntos internos del país, estas actitudes están cambiando en la medida en que los intereses de México están creciendo en Canadá. 
Los aspectos sobre la seguridad económica relacionados con el TLCAN no han escapado también a las críticas en ambos países, especialmente en México, al igual que el vínculo entre los aspectos ambientales y la seguridad. Canadá sabe bien de las implicaciones en materia de seguridad de una probable inmigración masiva y descontrolada proveniente de México, pero parece haber opiniones muy diversas sobre este complejo problema.

Por otro lado, existe una total diferencia sobre otros aspectos de seguridad. Canadá y México, por ejemplo, no comparten la misma visión con respecto a los cambios que se requieren en los acuerdos existentes sobre la seguridad interamericana. Tampoco coinciden con respecto al contexto y responsabilidades futuras de la Junta Interamericana de Defensa. Por lo tanto, se puede establecer que, hoy en día, existen muchos temas en el amplio y definido campo de la seguridad en que los dos países coinciden, pero también están presentes temas fundamentales que los separan, como históricamente ha ocurrido en el terreno militar, en los que Canadá, por razones geopolíticas y estratégicas, se ha alineado a las políticas de Estados Unidos.

A partir de sus experiencias históricas muy diferentes, los dos países han visto que tienen mucho más en común que en el pasado. Aún en los tiempos difíciles de finales del reciente siglo pasado, quedó en claro que existe un gran potencial en la combinación de esfuerzos en muchas áreas de interés común. Varios avances se han logrado ya económica y políticamente y, a pesar de uno que otro revés en materia de seguridad, el futuro parece promisorio para una relación mucho más fructífera entre Canadá y México.

\section{Fuente}

Con base en H.P. Klepak, ¿NATURAL ALLIES?. Canadian and Mexican Perspectives on International Security. Carleton University Press and Canadian Foundation for the Americas, Canadá, 1996. 